\title{
RESEARCH
}

Open Access

\section{Neighborhood food environment and mortality among older Japanese adults: results from the JAGES cohort study}

Yukako Tani ${ }^{1,2^{*}}$ (D, Norimichi Suzuki ${ }^{3}$, Takeo Fujiwara ${ }^{1}$, Masamichi Hanazato ${ }^{3}$, Naoki Kondo ${ }^{4}$, Yasuhiro Miyaguni ${ }^{5}$ and Katsunori Kondo 5,6

\begin{abstract}
Background: Previous research has linked lower availability of food stores selling fruits and vegetables to unhealthy diet. However, the longitudinal association between the availability of healthy food stores and mortality is unknown. This study examined the association between neighborhood availability of food stores and mortality by driving status among older adults.

Methods: This study drew upon a three-year follow up of participants in the Japan Gerontological Evaluation Study, a population-based cohort study of Japanese adults aged 65 years or older. Mortality from 2010 to 2013 was analyzed for 49,511 respondents. Neighborhood availability of food stores was defined as the number of food stores selling fruits and vegetables within a 500-m or 1-km radius of a person's residence. Both subjective (participant-reported) and objective (geographic information system-based) measurements were used to assess this variable. Cox regression models were used to estimate hazard ratios (HR) for mortality.

Results: A total of 2049 deaths occurred during the follow up. Lower subjective availability of food stores was significantly associated with increased mortality. Compared with participants reporting the highest availability, the age- and sex-adjusted HR for those reporting the lowest availability was 1.28 (95\% Cl: 1.04-1.58; $p=0.02$ ). The association remained significant after adjustment for sociodemographic (education, income, cohabitation, marital status, and employment status) and environmental (driving status, use of public transportation, and study site) status $(H R=1.24,95 \% \mathrm{Cl}: 1.01-1.53, p=0.04)$. This association was stronger among non-car users, among whom the HR for those reporting the lowest availability of food stores was 1.61 (95\% Cl: 1.08-2.41, $p=0.02$ ). In contrast, no significant association was seen between objective availability and mortality.

Conclusions: Lower availability of healthy food stores measured subjectively, but not objectively, was associated with mortality, especially among non-car users. Considering the decline in mobility with age, living in a neighborhood with many options for procuring fruits and vegetables within walking distance may be important for healthy aging.
\end{abstract}

Keywords: Food environment, Food store availability, Mortality, Older adults, Japan

\footnotetext{
* Correspondence: tani.hlth@tmd.ac.jp

'Department of Global Health Promotion, Tokyo Medical and Dental

University, 1-5-45, Yushima, Bunkyo-ku, Tokyo 113-8519, Japan

${ }^{2} J a p a n$ Society for the Promotion of Science, 5-3-1, Kojimachi, Chiyoda-ku,

Tokyo 102-0083, Japan

Full list of author information is available at the end of the article
}

(c) The Author(s). 2018 Open Access This article is distributed under the terms of the Creative Commons Attribution 4.0 International License (http://creativecommons.org/licenses/by/4.0/), which permits unrestricted use, distribution, and reproduction in any medium, provided you give appropriate credit to the original author(s) and the source, provide a link to the Creative Commons license, and indicate if changes were made. The Creative Commons Public Domain Dedication waiver (http://creativecommons.org/publicdomain/zero/1.0/) applies to the data made available in this article, unless otherwise stated. 


\section{Background}

Population aging is a global trend. In Japan, the proportion of people aged $\geq 60$ years is the highest in the world, exceeding 30\% [1]. This context can help to elucidate the secret of longevity. Along with its aging population, Japan's family structures have also changed. Multigenerational living arrangements are becoming less common, and the majority of older people lives alone or with their spouses [2]. Thus, older adults are faced with the task of obtaining food on their own.

Several food-related environmental factors influence the ability to obtain healthy food; these factors include availability (adequacy of a healthy food supply-e.g., the number of food stores that sell healthy food near people's homes), accessibility (travel time and distance), type and size of food stores, variety of items, price, and quality of food items [3, 4]. Among these factors, availability and accessibility are crucial for older people, because mobility (e.g., walking, driving a car, and using public transport) declines with age [5]. This decline affects older people's diets and health outcomes [5-7]. However, most previous studies on food availability have been cross-sectional and, with the exception of studies in the United Kingdom, conducted in areas of low population density and therefore in environments with a low density of food stores (e.g., the United States (US), Australia, New Zealand, and Canada) [4, 8]. Population density, which takes into account both population and land area, is approximately one to two orders of magnitude higher in Japan than in these previous studies' sites, with a population density of 348 people $/ \mathrm{km}^{2}$ in Japan, compared with 35 people $/ \mathrm{km}^{2}$ in the US, 0.3 people $/ \mathrm{km}^{2}$ in Australia, 17 people $/ \mathrm{km}^{2}$ in New Zealand, and 4 people $/ \mathrm{km}^{2}$ in Canada [9]. The impact of food availability on health in Japan and other high-density food store environments remains unclear.

Furthermore, few food availability studies have focused on older adults [7, 10-12]. One intervention study showed that a mobile catering van that carried fresh food to housing sites for low-income seniors increased vegetable intake among older adults [13]. Beyond the association with diet, several studies have reported that limited availability of healthy food increases the risks of obesity and diabetes [14-16]. However, to our knowledge, no study has investigated the impact of the availability of healthy food on mortality. Moreover, previous studies have failed to account for mobility status.

To adequately evaluate the association between the availability of food stores and mortality, it is important to account for differences in measurement-the objective or subjective measurement of the availability of food stores. Objective measurement uses geographic information systems (GIS) to assess the exact number of food stores within a given distance. Subjective (perceived) measurement uses questionnaires to assess an individuals' perceptions of food store availability in their neighborhoods [17-19]. Although most previous studies used objective measurements of availability $[17,18]$, a systematic review evaluating the association of objective and subjective measures of food store environments with dietary outcomes showed more consistent significant relationships for subjective measures than for objective measures [4]. We thus assessed both objective and subjective measures of food store availability in the present study.

We examined the impact of subjective and objective food store availability on mortality risk by driving status, using data from the Japan Gerontological Evaluation Study (JAGES), which is a large-scale, population-based cohort study of older people in Japan [20, 21]. We considered driving status an important variable because it was hypothesized that the effect of food store availability on mortality differs by mobility status.

\section{Methods \\ Study design and participants}

JAGES is a prospective cohort study established in 2010 to evaluate the social determinants of healthy aging among non-disabled older adults in Japan. This research drew on JAGES data from 15 municipalities in five prefectures (Hokkaido, Aomori, Yamanashi, Aichi, and Nagasaki). The baseline survey was conducted from August 2010 to December 2011. Self-administered questionnaires were distributed by mail to 86,967 people aged $\geq 65$ years and who were physically and cognitively independent, were not eligible for benefits from the public long-term care insurance program, and lived independently in the community. The survey was conducted using random sampling in eight large municipalities and was administered to all eligible residents in seven small municipalities. A total of 56,910 questionnaires were returned (65\% response rate), and $98 \%$ of them have been linked to death records $(N=55,510)$. The present analyses were carried out using data for 49,511 participants. We excluded respondents with missing data for residential address $(N=465)$ or sex $(N=2)$, and those who answered "unknown" $(N=710)$ or provided no answer $(N=2475)$ on the question about the subjective availability of food stores. Additionally, to ensure that the analytical sample included only participants who were actually physically and cognitively independent, we also excluded those who reported limitations $(N=983)$ or provided no answer $(N=1364)$ on the questions about activities of daily living (defined as being unable to walk, take a bath, or use the toilet without assistance [22] - these respondents were included in the JAGES accidentally). JAGES participants were informed that their participation in the study was voluntary and that 
completing and returning the questionnaire by mail indicated their consent to participate. The study protocol was approved by the Ethics Committees in the Research of Human Subjects at Nihon Fukushi University (№ 1005) and Chiba University Faculty of Medicine (№ 1777).

\section{Mortality outcome}

We determined vital status during the follow-up period from 2010 to 2013 (mean: 2.9 years; range: 2.1-3.5 years) by linking the cohort participants to mortality records in the national long-term care insurance database. There were a total of 2049 deaths in the analytical sample (cumulative mortality $=2049 / 49,511 ; 4.1 \%$ ). The present study examined all-cause mortality instead of cause-specific mortality because death certificate data were unavailable.

\section{Subjective availability of food stores}

Self-reported questionnaires were used to assess the subjective availability of food stores by asking, "How many stores or facilities selling fresh fruits and vegetables are located within 1 kilometer of your home?" Responses were given on a four-point Likert scale as follows: "many," "some," "few," or "none" [10]. These responses corresponded to highest, middle-high, middle-low, and lowest subjective food availability, respectively. The responses were used instead of specific numbers because participants' feelings of sufficiency or insufficiency may vary by neighborhood environment and social context.

\section{Objective availability of food stores}

The 500-meter $(\mathrm{m})$ mesh data from the Ministry of Economy, Trade and Industry Commerce Establishment Survey of 2007 [23] were used to assess the objective availability of food stores. We defined "food stores" as stores providing fresh fruits and vegetables. These included department stores, general merchandise stores, specialized supermarkets, and daily commodities stores. Because the association of food store availability with fruit and vegetable consumption varies by buffer distance [24], we calculated the number of stores along a straight line within a $500-\mathrm{m}$ and 1-kilometer $(\mathrm{km})$ radius of the center of participants' residential community blocks (chocho-aza, the smallest administrative unit in Japan, roughly comparable to a US census-block group [25]). Assuming all types of stores were equally distributed within the 500-m mesh, we calculated the number of stores along a straight line within a $500-\mathrm{m}$ and $1-\mathrm{km}$ radius of the participants' residences in accordance with the proportional distribution area of the map area (Additional file 1: Figure S1). ArcGIS 10.1 software was used for all spatial calculations. For the analyses, participants were categorized into quartiles based on the number of food stores within a $500-\mathrm{m}$ or $1-\mathrm{km}$ radius of their residence.

\section{Driving status}

Participants were asked whether they drive a car by themselves or travel in a family member's car when they go out. Driving status was classified as "non-car user" (for participants who neither drive a car by themselves nor travel in a family member's car) or "car user" (for participants who either drive a car by themselves or travel in a family member's car).

\section{Covariates}

Each municipality provided data on the participants' age at recruitment (65-69, 70-74, 75-79, or $\geq 80$ years) and sex. Sociodemographic status, use of public transportation, walking and going out, and nutritional and health status were assessed using a self-reported questionnaire. Sociodemographic status included education level $(\leq 9$, $10-12$, or $\geq 13$ years, or other), annual household income $(<2.00,2.00-3.99$, or $\geq 4.00$ million yen), living situation (lives with others or lives alone), marital status (married, widowed, divorced, never married, or other), and employment status (working, retired, or never worked) [26]. Annual household income was adjusted for household size, dividing the income by the square root of the number of people in the household. Environmental status included use of public transportation (train and bus) and the prefecture of residence (Hokkaido, Aomori, Yamanashi, Aichi, or Nagasaki). To determine use of public transportation, participants were asked whether they use a train and/or bus when they go out. Variables representing walking and going out included walking time $(\geq 30 \mathrm{~min} /$ day or $<30 \mathrm{~min} /$ day $)$ and frequency of going out ( $\geq 2$ times/week or $\leq 1$ time/ week). Nutritional status included body mass index (BMI) and frequency of fruit/vegetable intake ( $\geq 1$ time/ day or $<1$ time/day) and meat/fish intake ( $\geq 1$ time/day or $<1$ time/day) over the past month [10, 27]. We used standard categories of BMI to characterize participants as obese $\left(\geq 30.0 \mathrm{~kg} / \mathrm{m}^{2}\right)$, overweight $\left(25.0-29.9 \mathrm{~kg} / \mathrm{m}^{2}\right)$, normal $\left(18.5-24.9 \mathrm{~kg} / \mathrm{m}^{2}\right)$, or underweight $\left(<18.5 \mathrm{~kg} / \mathrm{m}^{2}\right)$ [28]. Although this categorization was not specifically developed for older adults, a Japanese cohort study reported that both $\mathrm{BMI}<18.5 \mathrm{~kg} / \mathrm{m}^{2}$ and $\geq 30 \mathrm{~kg} / \mathrm{m}^{2}$ were associated with increased risks of mortality among older adults [29]. Variables representing health status included medical treatment of diseases/symptoms and depressive symptoms. With multiple responses allowed, the participants were asked whether they were currently under medical treatment for cancer, heart disease, stroke, diabetes mellitus, respiratory diseases, or other diseases (hypertension, obesity, hyperlipidemia, osteoporosis, joint disease/ neuralgia, external injury/fracture, gastrointestinal diseases, liver diseases, psychiatric diseases, dysphagia, visual impairment, hearing impairment, elimination problems, sleep disorders, unidentified diseases, and other) [20]. The 
JAGES used the Japanese version of the Geriatric Depression Scale-15 (GDS) [30] to measure levels of depressive symptoms among older adults and classify them into two groups: non-depressed $(<5)$ or depressed $(\geq 5)[26,31]$. The population density of inhabitable land in participants' residential school districts was calculated using the 2010 census and land-utilization tertiary mesh data (as of 2010) from the Ministry of Land, Infrastructure, Transport and Tourism's National Land Numerical Information, based on a 1:25,000 topographic map of Japan. These calculations excluded non-developed areas (e.g., rivers, lakes, forests, and wasteland) [32]. Covariates with missing data were categorized as "missing."

\section{Statistical analysis}

Cox proportional hazards models were estimated, yielding hazard ratios (HR) and 95\% confidence intervals (CI) for all-cause mortality over the three-year follow-up period. The following sequence of models was constructed: Model 1 was adjusted for age and sex; Model 2 was additionally adjusted for sociodemographic status, with education, annual income, living situation, marital status, and employment status as potential confounders; Model 3 was further adjusted for environmental status, with driving status, public transportation (train and bus) use, and prefecture of residence as covariates, to examine whether the relationship between food store availability and mortality was independent of other environmental factors; Model 4 was also adjusted for walking and going out (walking time and frequency of going out) as potential mediating factors linking food store availability to mortality; and Model 5 was further adjusted for nutritional status (BMI, frequency of fruit/vegetable and meat/fish intake), health status (medical treatment for cancer, heart disease, stroke, diabetes mellitus, respiratory diseases, or other diseases), and depressive symptoms as potential mediating factors. The analysis was stratified by driving status because we found a significant interaction between driving status and food store availability (Additional file 1: Table S1). All analyses were conducted using Stata, Version 14. Participants with missing data on covariates were included in the analysis.

\section{Results}

Table 1 presents the baseline characteristics of the participants. Of all of the participants, $16.0 \%$ rated their subjective availability of food stores as "highest" and $5.4 \%$ rated it as "lowest." The objective measurement showed participants with a high subjective availability tended to live in neighborhoods with more food stores, compared with those with a low subjective availability. The mean number of food stores within a $500-\mathrm{m}$ radius of residence calculated by GIS (objective measure) was 12.3 for those who rated their subjective availability as "highest" and 5.5 for those who rated it as "lowest"
(Additional file 1: Table S2). The objective measurement showed mean numbers of food stores within a $500-\mathrm{m}$ radius of residence as $27,11,4$, and 0.8 for the fourth (highest), third, second, and first (lowest) quartiles, respectively. Of all car users, 16\% drove themselves and traveled in a family member's car, $52 \%$ drove themselves only, and $32 \%$ traveled in a family member's car only (data not shown in table). Non-car users also tended not to use public transportation; the percentages of both train and bus users among this group were less than $10 \%$ (Table 1). Most non-car users were categorized into the fourth quartile of objective availability of food stores, whereas most of car users were categorized into the first or second quartiles of objective availability of food stores. This means that non-car users tended to live in neighborhoods with many food stores, while car users tended to live in neighborhoods with few food stores.

Among all participants, the incidence rate of death per 100,000 person-years was 3.6 for participants with the highest subjective availability of food stores, compared with 4.4 for participants with the lowest subjective availability (Table 2). Regarding objective availability of food stores within a 500-m radius of residence, the incidence rate of death per 100,000 person-years was 3.7 for participants in the fourth (highest) quartile of food store availability, compared with 4.3 for participants in the first (lowest) quartile of availability. Table 3 shows the results of multivariate Cox regression models. After adjusting for age and sex, lower subjective availability was associated with mortality $(\mathrm{HR}=1.21,95 \% \mathrm{CI}: 1.04-$ 1.41 for middle-low subjective availability and $\mathrm{HR}=$ 1.28, 95\% CI: $1.04-1.58$ for lowest subjective availability; $p$ for trend < 0.01) (Table 3, Model 1). After further adjusting for sociodemographic and environmental status, these HRs were attenuated but remained statistically significant (HR $=1.19,95 \%$ CI: $1.02-1.39$ for middle-low subjective availability and $\mathrm{HR}=1.24,95 \% \mathrm{CI}: 1.01-1.53$ for lowest subjective availability; $p$ for trend $=0.01$ ) (Model 3). After adjusting for potential mediators (walking and going out, nutritional and health status), these HRs diminished and became statistically non-significant (Models 4 and 5). In contrast, there was no significant association between the objective availability of food stores in a 500-m radius and mortality. When we conducted the analysis using a $1-\mathrm{km}$ radius instead of a 500-m radius, we still observed no significant association for the objective measurement of food store availability (data not shown in table).

The interaction between the subjective availability of food stores and driving status was significant, indicating that the effect of the subjective availability of food stores on mortality was attenuated when participants used cars (Additional file 1: Table S1). When stratified by driving status, the incidence rate of death per 100,000 
Table 1 Baseline characteristics of older Japanese adults by driving status ( $n=49,511)$

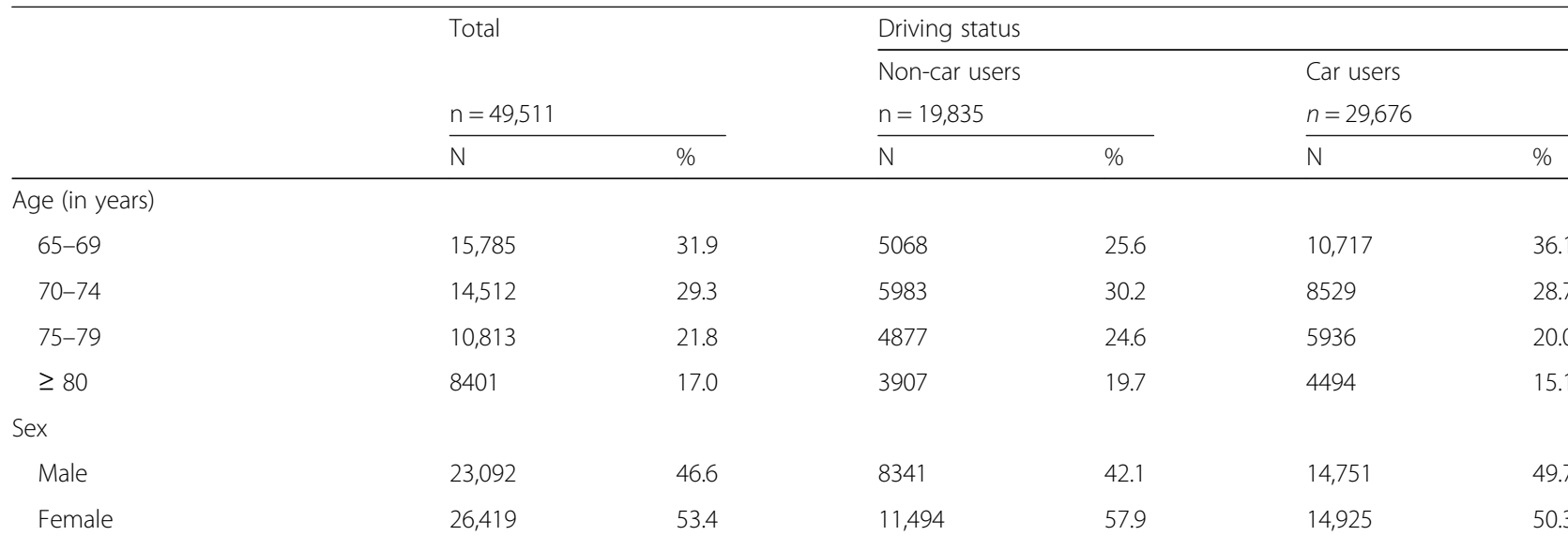

Food store availability

Subjective (perceived number of food stores within 1-km of residence)

$\begin{array}{lllllll}\text { Highest } & 7898 & 16.0 & 3359 & 16.9 & 4539 & 15.3 \\ \text { Middle-high } & 30,013 & 60.6 & 12,102 & 61.0 & 17,911 & 60.4 \\ \text { Middle-low } & 8935 & 18.0 & 3565 & 18.0 & 5370 & 18.1 \\ \text { Lowest } & 2665 & 5.4 & 809 & 4.1 & 1856\end{array}$

Objective (mean number of food stores within 500-m radius of residence)

$\begin{array}{lll}\text { Quartile } 4(27) & 12,375 & 25.0 \\ \text { Quartile } 3(11) & 12,010 & 24.3 \\ \text { Quartile 2 (4) } & 12,685 & 25.6 \\ \text { Quartile } 1(0.8) & 12,441 & 25.1\end{array}$

Objective (mean number of food stores within 1-km radius of residence)

$\begin{array}{lll}\text { Quartile } 4(91) & 12,369 & 25.0 \\ \text { Quartile } 3(36) & 12,370 & 25.0 \\ \text { Quartile 2 (16) } & 12,378 & 25.0 \\ \text { Quartile 1 (4) } & 12,394 & 25.0\end{array}$

Sociodemographic status

Education (in years)

\begin{tabular}{|c|c|c|c|c|c|c|}
\hline Low $(\leq 9)$ & 22,736 & 45.9 & 8311 & 41.9 & 14,425 & 48.6 \\
\hline Middle (10-12) & 16,250 & 32.8 & 6477 & 32.7 & 9773 & 32.9 \\
\hline High ( $\geq 13$ ) & 8147 & 16.5 & 3794 & 19.1 & 4353 & 14.7 \\
\hline Other/missing & 2378 & 4.8 & 1253 & 6.3 & 1125 & 3.8 \\
\hline \multicolumn{7}{|c|}{ nnual income (in millions of yen) } \\
\hline $\operatorname{Low}(<2.00)$ & 20,101 & 40.6 & 7898 & 39.8 & 12,203 & 41.1 \\
\hline Middle (2.00-3.99) & 15,636 & 31.6 & 5856 & 29.5 & 9780 & 33.0 \\
\hline High ( $\geq 4.00)$ & 4496 & 9.1 & 1759 & 8.9 & 2737 & 9.2 \\
\hline Missing & 9278 & 18.7 & 4322 & 21.8 & 4956 & 16.7 \\
\hline \multicolumn{7}{|l|}{ iving situation } \\
\hline Lives with others & 41,237 & 83.3 & 15,006 & 75.7 & 26,231 & 88.4 \\
\hline Lives alone & 6810 & 13.8 & 4070 & 20.5 & 2740 & 9.2 \\
\hline Missing & 1464 & 3.0 & 759 & 3.8 & 705 & 2.4 \\
\hline \multicolumn{7}{|l|}{ Marital status } \\
\hline Married & 34,309 & 69.3 & 12,157 & 61.3 & 22,152 & 74.6 \\
\hline
\end{tabular}


Table 1 Baseline characteristics of older Japanese adults by driving status ( $n=49,511)$ (Continued)

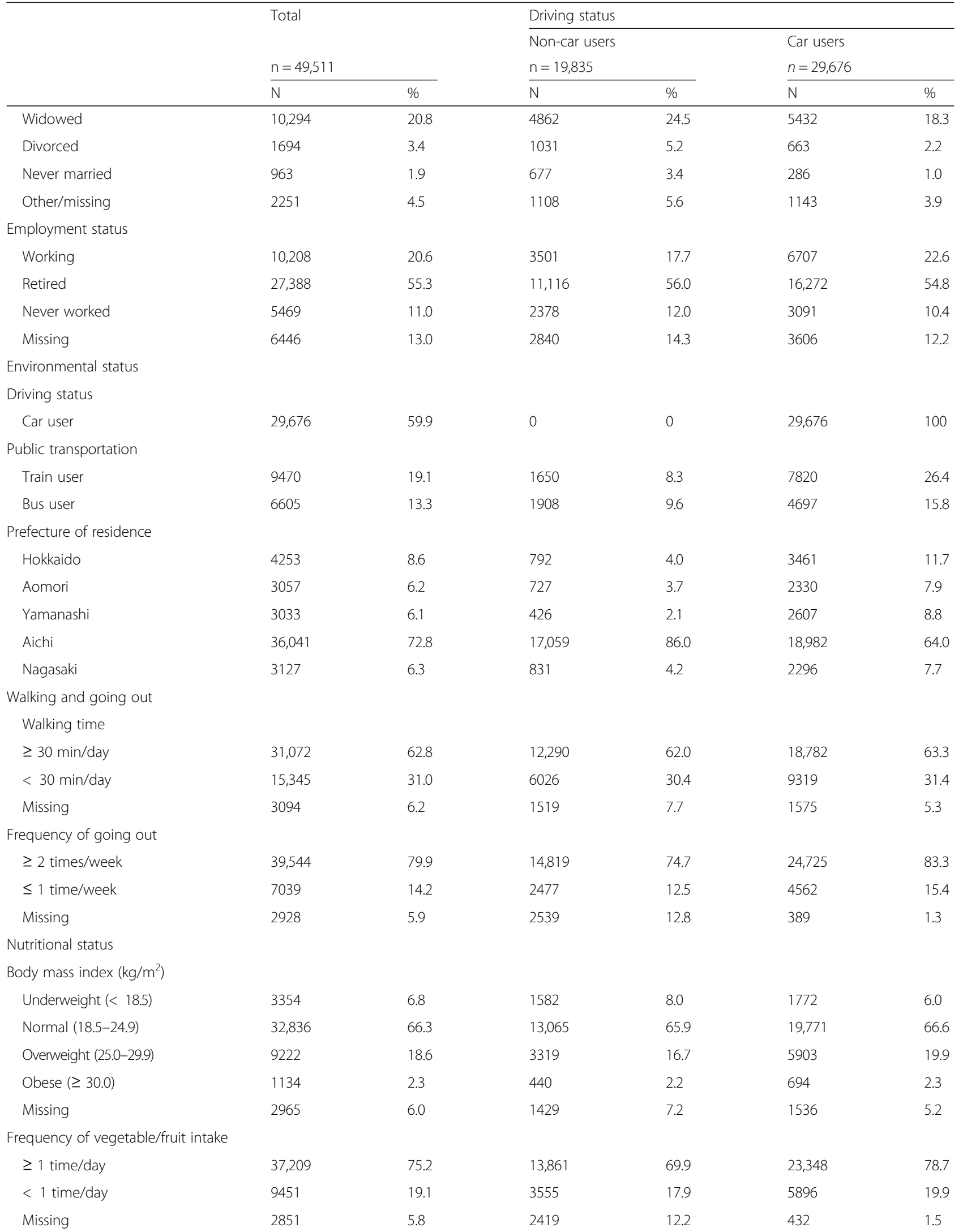


Table 1 Baseline characteristics of older Japanese adults by driving status ( $n=49,511)$ (Continued)

\begin{tabular}{|c|c|c|c|c|c|c|}
\hline & \multicolumn{2}{|l|}{ Total } & \multicolumn{4}{|c|}{ Driving status } \\
\hline & & & Non-ca & & Car use & \\
\hline & \multicolumn{2}{|c|}{$n=49,511$} & \multicolumn{2}{|c|}{$n=19,835$} & \multicolumn{2}{|c|}{$n=29,676$} \\
\hline & $\mathrm{N}$ & $\%$ & $\mathrm{~N}$ & $\%$ & $\mathrm{~N}$ & $\%$ \\
\hline \multicolumn{7}{|l|}{ Frequency of meat/fish intake } \\
\hline$\geq 1$ time/day & 19,282 & 38.9 & 7284 & 36.7 & 11,998 & 40.4 \\
\hline$<1$ time/day & 27,094 & 54.7 & 9980 & 50.3 & 17,114 & 57.7 \\
\hline Missing & 3135 & 6.3 & 2571 & 13 & 564 & 1.9 \\
\hline \multicolumn{7}{|l|}{ Health status } \\
\hline \multicolumn{7}{|l|}{ Undergoing medical treatment } \\
\hline Cancer (yes) & 2134 & 4.3 & 903 & 4.6 & 1231 & 4.1 \\
\hline Heart disease (yes) & 5983 & 12.1 & 2390 & 12.0 & 3593 & 12.1 \\
\hline Stroke (yes) & 669 & 1.4 & 251 & 1.3 & 418 & 1.4 \\
\hline Diabetes mellitus (yes) & 6247 & 12.6 & 2448 & 12.3 & 3799 & 12.8 \\
\hline Respiratory disease (yes) & 1795 & 3.6 & 673 & 3.4 & 1122 & 3.8 \\
\hline Other (yes) & 34,210 & 69.1 & 13,878 & 70.0 & 20,332 & 68.5 \\
\hline \multicolumn{7}{|l|}{ Depressive symptoms } \\
\hline $\begin{array}{l}\text { Non-depressed } \\
(G D S<5)\end{array}$ & 29,877 & 60.3 & 11,264 & 56.8 & 18,613 & 62.7 \\
\hline Depressed (GDS $\geq 5$ ) & 11,365 & 23.0 & 4656 & 23.5 & 6709 & 22.6 \\
\hline Missing & 8269 & 16.7 & 3915 & 19.7 & 4354 & 14.7 \\
\hline
\end{tabular}

GDS $=$ Geriatric Depression Scale

person-years was 2.7 for participants with the highest subjective availability and 4.5 for those with the lowest subjective measurement among non-car users, compared with an incidence rates of death of 4.1 for participants with the highest subjective availability and 4.3 for participants with the lowest subjective availability among car users (Table 2). Table 4 demonstrates that lower subjective availability was associated with mortality among non-car users. Compared with the highest subjective availability of food stores, the HR adjusting for age and sex was 1.46 (95\% CI: 1.15-1.86) for middle-high subjective availability, 1.64 (95\% CI: 1.24-2.17) for middlelow subjective availability, and 1.61 (95\% CI: $1.08-2.41)$ for lowest subjective availability ( $p$ for trend <0.01) (Model 1). After further adjusting for sociodemographic and environmental status, the HRs were attenuated but remained statistically significant, except that for lowest subjective availability $(\mathrm{HR}=1.41,95 \% \mathrm{CI}$ : $1.11-1.80$ for middle-high; $\mathrm{HR}=1.55$, 95\% CI: $1.17-2.05$ for middlelow; and $\mathrm{HR}=1.44,95 \% \mathrm{CI}: 0.96-2.16$ for lowest subjective availability; $p$ for trend $=0.01$ ) (Model 3 ). This association remained significant even after adjusting for potential mediators (Models 4 and 5). In contrast to non-car users, no significant association between subjective availability and mortality was found among car users. Objective availability was not associated with mortality among those of either driving status.

\section{Discussion}

To our knowledge, this was the first study to examine the association between the availability of healthy food stores and mortality using both subjective and objective availability measurements. Among all participants, we found that lower subjective, but not objective, availability of food stores was associated with mortality. This association persisted after adjusting for age, sex, sociodemographic characteristics, and environmental status; however, it became statistically non-significant after adjusting for walking and going out, nutritional status, and health status. When stratified by driving status, we found that lower subjective availability of food stores was associated with mortality among non-car users, but not car users. This association was attenuated, but remained significant, even after adjusting for walking and going out, nutritional status, and health status.

This study described the effect of subjective availability in the context of Japan's considerably higher population density and higher density of food stores, compared with the previous study settings of the US, Australia, New Zealand, and Canada. In our study sites in Japan, a mean of 10.6 food stores were found to be within a 500-m radius of a participant's residence, and the mean population density of inhabitable land [32] was 3674 people/ $\mathrm{km}^{2}$. Our findings suggest that living in a neighborhood with many options for accessing fruits and vegetables, 
Table 2 All-cause mortality by driving status among older Japanese adults ( $n=49,511)$ during the 3-year follow-up period

\begin{tabular}{|c|c|c|c|c|c|c|}
\hline & \multirow{2}{*}{\multicolumn{2}{|c|}{ Total $(n=49,511)$}} & \multicolumn{4}{|c|}{ Driving status } \\
\hline & & & \multicolumn{2}{|c|}{ Non-car users $(n=19,835)$} & \multicolumn{2}{|c|}{ Car users $(n=29,676)$} \\
\hline & $\begin{array}{l}\text { Number of } \\
\text { deaths (\%) }\end{array}$ & $\begin{array}{l}\text { Incidence rate per } \\
100,000 \text { person- } \\
\text { years }(95 \% \mathrm{Cl})\end{array}$ & $\begin{array}{l}\text { Number of } \\
\text { deaths (\%) }\end{array}$ & $\begin{array}{l}\text { Incidence rate per } \\
\text { 100,000 person- } \\
\text { years }(95 \% \mathrm{Cl})\end{array}$ & $\begin{array}{l}\text { Number of } \\
\text { deaths (\%) }\end{array}$ & $\begin{array}{l}\text { Incidence rate per } \\
\text { 100,000 person- } \\
\text { years (95\% Cl) }\end{array}$ \\
\hline \multicolumn{7}{|l|}{ Food store availability } \\
\hline \multicolumn{7}{|l|}{ Subjective availability } \\
\hline Highest & $291(3.7)$ & $3.6(3.2-4.0)$ & $77(2.3)$ & $2.7(2.1-3.4)$ & $214(4.7)$ & $4.1(3.6-4.7)$ \\
\hline Middle-high & $1235(4.1)$ & $3.9(3.7-4.2)$ & $424(3.5)$ & $4.0(3.6-4.4)$ & $811(4.5)$ & $3.9(3.6-4.2)$ \\
\hline Middle-low & $396(4.4)$ & $4.2(3.8-4.6)$ & $144(4.0)$ & $4.5(3.8-5.3)$ & $252(4.7)$ & $4.1(3.6-4.6)$ \\
\hline Lowest & $127(4.8)$ & $4.4(3.7-5.2)$ & $35(4.3)$ & $4.5(3.3-6.3)$ & $92(5.0)$ & $4.3(3.5-5.3)$ \\
\hline \multicolumn{7}{|c|}{ Objective availability within a 500-meter radius of residence } \\
\hline Quartile 4 (highest) & $410(3.3)$ & $3.7(3.4-4.1)$ & $234(2.7)$ & $3.4(3.0-3.9)$ & $176(4.8)$ & $4.2(3.7-4.9)$ \\
\hline Quartile 3 & $462(3.8)$ & $3.8(3.4-4.1)$ & $183(3.3)$ & $3.8(3.3-4.4)$ & $279(4.3)$ & $3.8(3.3-4.2)$ \\
\hline Quartile 2 & $564(4.4)$ & $3.9(3.6-4.3)$ & $133(4.1)$ & $4.1(3.4-4.8)$ & $431(4.6)$ & $3.9(3.5-4.3)$ \\
\hline Quartile 1 (lowest) & $613(4.9)$ & $4.3(4.0-4.7)$ & $130(5.5)$ & $5.1(4.3-6.1)$ & $483(4.8)$ & $4.1(3.8-4.5)$ \\
\hline \multicolumn{7}{|c|}{ Objective availability within a 1-kilometer radius of residence } \\
\hline Quartile 4 (highest) & $360(2.9)$ & $3.4(3.1-3.8)$ & $244(2.5)$ & $3.3(2.9-3.7)$ & $116(4.2)$ & $3.8(3.2-4.6)$ \\
\hline Quartile 3 & $485(3.9)$ & $3.7(3.4-4.1)$ & $170(3.2)$ & $3.6(3.1-4.2)$ & $315(4.5)$ & $3.8(3.4-4.3)$ \\
\hline Quartile 2 & $559(4.5)$ & $4.0(3.6-4.3)$ & $135(5.2)$ & $4.8(4.1-5.7)$ & $424(4.3)$ & $3.7(3.4-4.1)$ \\
\hline Quartile 1 (lowest) & $645(5.2)$ & $4.5(4.2-4.9)$ & $131(5.7)$ & $5.2(4.4-6.2)$ & $514(5.1)$ & $4.4(4.0-4.8)$ \\
\hline
\end{tabular}

Cl confidence interval

Table 3 Hazard ratios with 95\% confidence intervals for the association of mortality with subjective and objective availability of food stores among older Japanese adults $(n=49,511)$

\begin{tabular}{|c|c|c|c|c|c|}
\hline & Model 1 & Model 2 & Model 3 & Model 4 & Model 5 \\
\hline & HR $(95 \% \mathrm{Cl})$ & HR (95\% Cl) & HR $(95 \% \mathrm{Cl})$ & HR $(95 \%$ Cl) & HR (95\% Cl) \\
\hline \multicolumn{6}{|l|}{ Food store availability } \\
\hline \multicolumn{6}{|l|}{ Subjective availability } \\
\hline Highest & ref & ref & ref & ref & ref \\
\hline Middle-high & $1.12(0.99-1.27)$ & $1.11(0.98-1.26)$ & $1.12(0.99-1.28)$ & $1.09(0.95-1.23)$ & $1.09(0.95-1.23)$ \\
\hline Middle-low & $1.21(1.04-1.41)^{*}$ & $1.18(1.01-1.37)^{*}$ & $1.19(1.02-1.39)^{*}$ & $1.11(0.95-1.29)$ & $1.05(0.90-1.23)$ \\
\hline Lowest & $1.28(1.04-1.58)^{*}$ & $1.25(1.01-1.54)^{*}$ & $1.24(1.01-1.53)^{*}$ & $1.13(0.91-1.39)$ & $1.05(0.85-1.30)$ \\
\hline$p$ for trend & $<0.01$ & 0.02 & 0.01 & 0.19 & 0.74 \\
\hline \multicolumn{6}{|c|}{ Objective availability within a 500-meter radius of residence } \\
\hline Quartile 4 (highest) & ref & ref & ref & ref & ref \\
\hline Quartile 3 & $0.99(0.87-1.14)$ & $0.98(0.86-1.12)$ & $0.98(0.86-1.12)$ & $0.96(0.84-1.10)$ & $0.95(0.83-1.10)$ \\
\hline Quartile 2 & $1.05(0.93-1.20)$ & $1.04(0.91-1.18)$ & $1.04(0.91-1.19)$ & $1.01(0.88-1.15)$ & $1.01(0.89-1.16)$ \\
\hline Quartile 1 (lowest) & $1.10(0.97-1.25)$ & $1.07(0.94-1.22)$ & $1.05(0.92-1.21)$ & $1.00(0.87-1.15)$ & $1.01(0.88-1.16)$ \\
\hline$p$ for trend & 0.08 & 0.18 & 0.31 & 0.75 & 0.58 \\
\hline
\end{tabular}

$H R$ hazard ratio, $\mathrm{Cl}$ confidence interval, ref reference group

Model 1: Adjusted for age and sex

Model 2: Model $1+$ adjusted for sociodemographic status (education, annual income, living situation, marital status, and employment status)

Model 3: Model $2+$ adjusted for environmental status (driving status, public transportation [train and bus], and prefecture of residence)

Model 4: Model 3 + adjusted for walking and going out (walking time and frequency of going out)

Model 5: Model 4 + adjusted for nutritional status (body mass index, frequency of fruit/vegetable and meat/fish intake) and health status (medical treatment for cancer, heart disease, stroke, diabetes mellitus, respiratory diseases, or other, and depressive symptoms)

${ }^{*} p<0.05$ 
Table 4 Hazard ratios with 95\% confidence intervals for the association of mortality with subjective and objective availability of food stores by driving status among older Japanese adults

\begin{tabular}{|c|c|c|c|c|c|}
\hline & Model 1 & Model 2 & Model 3 & Model 4 & Model 5 \\
\hline & HR $(95 \% \mathrm{Cl})$ & HR $(95 \% \mathrm{Cl})$ & HR $(95 \% \mathrm{Cl})$ & HR (95\% Cl) & HR $(95 \% \mathrm{Cl})$ \\
\hline \multicolumn{6}{|l|}{ Driving status } \\
\hline \multicolumn{6}{|c|}{ Non-car users $(n=19,835)$} \\
\hline \multicolumn{6}{|l|}{ Subjective availability } \\
\hline Highest & ref & ref & ref & ref & ref \\
\hline Middle-high & $1.46(1.15-1.86)^{* *}$ & $1.44(1.13-1.83)^{* *}$ & $1.41(1.11-1.80)^{* *}$ & $1.36(1.06-1.73)^{*}$ & $1.38(1.08-1.76)^{*}$ \\
\hline Middle-low & $1.64(1.24-2.17)^{* * *}$ & $1.59(1.20-2.10)^{* *}$ & $1.55(1.17-2.05)^{* *}$ & $1.43(1.08-1.89)^{*}$ & $1.37(1.03-1.82)^{*}$ \\
\hline Lowest & $1.61(1.08-2.41)^{*}$ & $1.56(1.04-2.33)^{*}$ & $1.44(0.96-2.16)$ & $1.28(0.85-1.92)$ & $1.21(0.80-1.82)$ \\
\hline$p$ for trend & $<0.01$ & $<0.01$ & 0.01 & 0.08 & 0.22 \\
\hline \multicolumn{6}{|c|}{ Objective availability within a 500-meter radius of residence } \\
\hline Quartile 4 (highest) & ref & ref & ref & ref & ref \\
\hline Quartile 3 & $1.06(0.87-1.28)$ & $1.04(0.86-1.27)$ & $1.00(0.82-1.22)$ & $0.98(0.80-1.20)$ & $0.98(0.81-1.20)$ \\
\hline Quartile 2 & $1.06(0.85-1.32)$ & $1.04(0.84-1.30)$ & $0.99(0.79-1.24)$ & $0.95(0.76-1.19)$ & $0.92(0.73-1.15)$ \\
\hline Quartile 1 (lowest) & $1.19(0.95-1.48)$ & $1.15(0.91-1.44)$ & $0.99(0.78-1.26)$ & $0.92(0.72-1.18)$ & $0.97(0.76-1.24)$ \\
\hline$p$ for trend & 0.17 & 0.28 & 0.93 & 0.49 & 0.64 \\
\hline \multicolumn{6}{|l|}{ Car users $(n=29,676)$} \\
\hline \multicolumn{6}{|l|}{ Subjective availability } \\
\hline Highest & ref & ref & ref & ref & ref \\
\hline Middle-high & $0.99(0.86-1.16)$ & $0.99(0.85-1.15)$ & $1.01(0.86-1.17)$ & $0.97(0.84-1.13)$ & $0.96(0.83-1.12)$ \\
\hline Middle-low & $1.05(0.87-1.26)$ & $1.02(0.85-1.23)$ & $1.03(0.86-1.24)$ & $0.98(0.81-1.17)$ & $0.92(0.76-1.11)$ \\
\hline Lowest & $1.14(0.89-1.46)$ & $1.12(0.87-1.43)$ & $1.13(0.89-1.45)$ & $1.04(0.81-1.34)$ & $0.96(0.75-1.23)$ \\
\hline$p$ for trend & 0.24 & 0.39 & 0.34 & 0.85 & 0.49 \\
\hline \multicolumn{6}{|c|}{ Objective availability within a 500-meter radius of residence } \\
\hline Quartile 4 (highest) & ref & & & ref & ref \\
\hline Quartile 3 & $0.89(0.73-1.07)$ & $0.87(0.72-1.05)$ & $0.87(0.72-1.05)$ & $0.85(0.71-1.03)$ & $0.85(0.70-1.02)$ \\
\hline Quartile 2 & $0.95(0.80-1.14)$ & $0.93(0.78-1.11)$ & $0.93(0.78-1.11)$ & $0.91(0.77-1.09)$ & $0.94(0.79-1.13)$ \\
\hline Quartile 1 (lowest) & $0.98(0.82-1.16)$ & $0.95(0.80-1.13)$ & $0.95(0.79-1.13)$ & $0.92(0.77-1.10)$ & $0.93(0.78-1.11)$ \\
\hline$p$ for trend & 0.69 & 0.94 & 0.92 & 0.84 & 0.95 \\
\hline
\end{tabular}

$H R$ hazard ratio, $\mathrm{Cl}$ confidence interval; ref = reference group

Model 1: Adjusted for age and sex

Model 2: Model 1 + adjusted for sociodemographic status (education, annual income, living situation, marital status, and employment status)

Model 3: Model $2+$ adjusted for environmental status (public transportation (train and bus) and prefecture of residence)

Model 4: Model 3 + adjusted for walking and going out (walking time and frequency of going out)

Model 5: Model 4 + adjusted for nutritional status (body mass index, frequency of fruit/vegetable and meat/fish intake) and health status (medical treatment for cancer, heart disease, stroke, diabetes mellitus, respiratory diseases, or other, and depressive symptoms)

${ }^{*} p<0.05 ;{ }^{* *} p<0.01 ;{ }^{* * *} p<0.001$

and having a perception of high food availability, may be important for healthy aging in Japan.

We found that subjective, not objective, availability was associated with mortality. This pattern was previously observed in a systematic review evaluating the relationship between the availability of food stores and diet: Subjective measurements were found to be more consistently related to healthy dietary outcomes than were objective measurements [4]. In addition, we found that the associations between subjective availability measurements and mortality were significant and largely similar after adjusting for population density (Additional file 1: Table S3), suggesting that this relationship was independent of population density. Regarding objective availability, because of differences in food store densities across the study sites (prefectures), participants from sites with high-store density were overrepresented in the highest quartile of the objective measure. Therefore, we also conducted the analyses separately by study site using site-specific categories (based on within-site distributions). This analysis yielded similar results, suggesting 
the non-significant relationship was independent of study site (data not shown in table).

There are three possible explanations for this pattern. First, subjective availability incorporated realized access to food stores in the local vicinity, whereas objective availability simply indicated potential access [33]. Food choice is influenced by both potential access (e.g., number, size, and location of food stores) and realized access (e.g., neighborhood of residence, vehicle access, public transportation, and financial resources) [3]. Although our subjective measure of availability asked respondents to indicate the number of stores within $1 \mathrm{~km}$ of their home, the perception of this $1-\mathrm{km}$ radius could vary based on physical barriers in their area that are difficult to evaluate with GIS, including volume of street traffic or steep roads. We found that older adults who reported low subjective availability of food stores lived in hillier environments compared with those who reported high availability; the average land slope [32] was $4.5^{\circ}$ in areas with the lowest food store availability and $2.5^{\circ}$ in areas with the highest availability (Additional file 1: Table S4). For these reasons, the subjective measure may be more capable than the objective measure of accurately describing access to food stores. One way to account for realized access in the objective measure would be assessing road network buffers instead of circular buffers [18]. Further investigation using global positioning systems may elucidate the actual mechanism linking subjective, rather than objective, availability to mortality.

Second, the subjective measure of availability may capture a picture of relatively long-term food store availability. In contrast, the objective measure captures only a single time-point of food store availability. In Japan, the entry rate for food store businesses was around 15\% and the exit rate was around 30\% from 1997 to 2002 [34]. More than $90 \%$ of the participants had lived in the same municipality for $\geq 10$ years, and the subjective measure might be more representative of a participant's perception of long-term food store availability in their local area compared with the objective measurement of a single time-point. Future studies are needed to account for changes in the rates of the entry and exit of food store businesses, and for long-term effects.

Third, subjective measures may act as proxies for health consciousness. For example, older people with high levels of health consciousness may have detailed knowledge of food store availability in their neighborhood. If this is the case, raising health consciousness among older people is more important than increasing the number of food stores in setting with high density of food stores.

The impact of subjective availability was prominent among non-car users. This finding was plausible, as car users can shop outside their residential area. Because car use declines with age (less than half of older adults in Japan hold a driver's license, with $43 \%$ of adults aged $\geq 65$ years and $25 \%$ of adults aged $\geq 75$ years holding a license in 2010 [35]) and may be abandoned after a change in cohabitation, such as after the death of a spouse or partner, living in a neighborhood with many options for accessing fresh fruits and vegetables within walking distance may reduce the risk of mortality among older adults.

The association between the subjective availability of food stores and mortality was attenuated after adjusting for walking time and frequency of going out, which suggests neighborhood availability of food stores may contribute to reducing mortality risk by preventing physical inactivity. After retirement, grocery shopping may be a main reason for going out. Older adults who live in areas with a high availability of food stores within walking distance may frequently go out on foot for grocery shopping. In contrast, those who live in areas with a low availability of food stores may use a car or go out less frequently. In our sample, we found participants with low subjective availability of food stores tended to have shorter walking times and went out less frequently than did those with high subjective availability (Additional file 1: Table S5).

Key strengths of this study were its assessment of the effects of both subjective and objective availability of food stores on mortality, accounting for driving status, exploring these relationships in a high-density setting, and using a large-scale, population-based cohort study of older Japanese people. Several limitations of this study should be noted. First, we did not account for the type and size of food stores or for the price of food items; potentially important factors for making healthy food choices [3]. However, it is difficult to confirm the possible extent and direction of the resulting bias. Second, we did not account for availability of unhealthy food stores, such as fast food. Nonetheless, more than $90 \%$ of Japanese older adults reported that they visit fast-food restaurants less than once a month [36]. Third, our analysis was limited to all-cause mortality. Future studies should examine cause-specific mortality, especially diet-related outcomes, to clarify the effects of food store availability in the neighborhood. Fourth, we calculated objective availability using secondary data sources, which have been found to underestimate and overestimate food access, compared with primary data sources collected through field observation [37]. However, because it is difficult to collect data through field observation in population-based studies, we used government data, which are more reliable than are other secondary data. Fifth, we were unable to collect subjective and objective data for the same time period, which may have caused a difference in results for these measures. Sixth, we lacked 
information on the person responsible for grocery shopping and the frequency of car use, which may explain some of the variance in the association between food store availability and mortality. For example, even if the participant reported low subjective availability, the person responsible for grocery shopping in their household might report subjective availability as high, or vice versa. Finally, although the participants were exclusively physically and cognitively independent older people, we cannot rule out the possibility of an inverse association. For example, a person with a weak physical condition might move to an area that is convenient for shopping. However, more than $80 \%$ of the participants in this study had lived in the same municipality for $\geq 30$ years, and the results of the analyses limited to this subsample were generally similar to the results for the full sample (Additional file 1: Table S6). Additionally, the results were substantially similar when we excluded early deaths (within 1 year) from the analysis, (Additional file 1: Table S7).

\section{Conclusions}

Lower availability of healthy food stores, measured subjectively, but not objectively, was associated with mortality among non-car users, but not car users. Considering the decline of mobility with age, living in a neighborhood with many options for accessing fruits and vegetables within walking distance may be important for healthy aging. Future studies should address the mechanisms underlying the differences in these measures of food store availability. To design age-friendly neighborhoods and to secure access to healthy food, it is important to further investigate the factors influencing the subjective availability of food stores among older populations.

\section{Additional file}

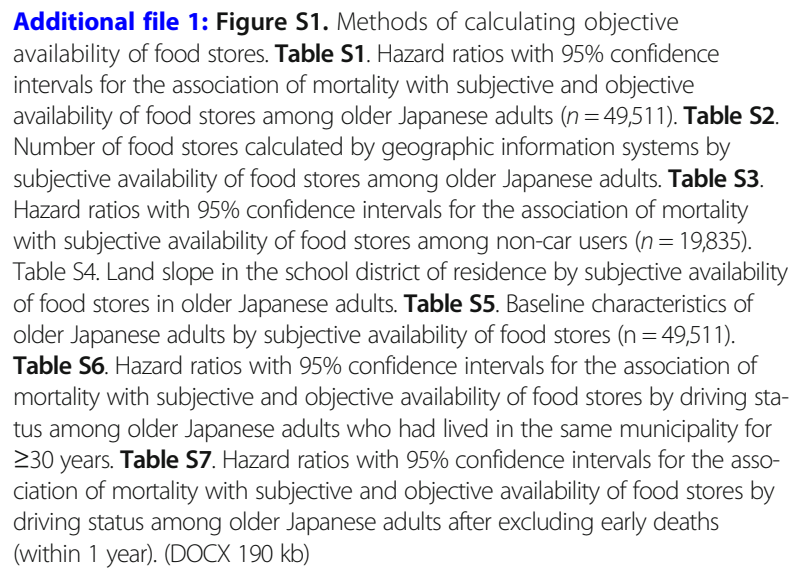

\section{Abbreviations}

95\% Cl: 95\% confidence interval; BMI: Body mass index; GDS: Geriatric Depression Scale; GIS: Geographic information system; HR: Hazard ratio;
JAGES: Japan Gerontological Evaluation Study; km: Kilometer; m: Meter; US: United States

\section{Acknowledgments}

We are particularly grateful to the staff members in each study area, and in the central office, for conducting the survey.

\section{Funding}

This work used data from JAGES, which was supported by the MEXT (Ministry of Education, Culture, Sports, Science and Technology, Japan) Supported Program for the Strategic Research Foundation at Private Universities (20092013); JSPS (Japan Society for the Promotion of Science) KAKENHI Grants (Numbers 22330172, 22390400, 23243070, 23590786, 23790710, 24390469, 24530698, 24683018, 25253052, 25870573, 25870881, 26285138, 26882010, and 15H01972); Health Labour Sciences Research Grants (Numbers H22-ChojuShitei-008, H24-Junkanki[Seishu]-Ippan-007, H24-Chikyukibo-Ippan-009, H24Choju-Wakate-009, H25-Kenki-Wakate-015, H25-Choju-Ippan-003, H26-IrryoShitei-003[Fukkou], H26-Choju-Ippan-006, H27-Ninchisyou-Ippan-001, and H28Choju-Ippan-002); Research and Development Grants for Longevity Science from the Japan Agency for Medical Research and Development; Research Funding for Longevity Sciences from the National Center for Geriatrics and Gerontology (Numbers 24-17 and 24-23); the Japan Foundation for Aging and Health (Number J09KF00804); and World Health Organization Centre for Health Development (WHO Kobe Centre) (WHO APW 2017/713981). This research also received funding from JSPS KAKENHI Grants (Numbers 16 K16295 and 16 J11423) and a Lotte Research Promotion Grant.

\section{Availability of data and materials}

The datasets used and analyzed for the current study are from the JAGES study. All inquiries are to be addressed to the data management committee via email: dataadmin.ml@jages.net. All JAGES datasets have ethical and legal restrictions for public deposition because of the inclusion of sensitive information on the human participants.

\section{Authors' contributions}

YT conceived of the design, analyzed the data, performed the literature review, and wrote the first draft of the article. NS, MH, NK, YM, and KK collected the data. TF revised the first draft. NS, MH, NK, YM, and KK edited the manuscript. All authors approved the final version of the manuscript.

\section{Ethics approval and consent to participate}

As we described in the Methods section, JAGES participants were informed that their participation in the study was voluntary and that completing and returning the questionnaire by mail indicated their consent to participate in the study. The study protocol was approved by the Ethics Committees in the Research of Human Subjects at Nihon Fukushi University (№ 10-05) and Chiba University Faculty of Medicine (№ 1777).

\section{Consent for publication}

Not applicable.

\section{Competing interests}

The authors declare that they have no competing interests.

\section{Publisher's Note}

Springer Nature remains neutral with regard to jurisdictional claims in published maps and institutional affiliations.

\section{Author details}

'Department of Global Health Promotion, Tokyo Medical and Dental University, 1-5-45, Yushima, Bunkyo-ku, Tokyo 113-8519, Japan. ${ }^{2} J a p a n$ Society for the Promotion of Science, 5-3-1, Kojimachi, Chiyoda-ku, Tokyo 102-0083, Japan. ${ }^{3}$ Center for Preventive Medical Sciences, Chiba University, 1-33 Yayoicho, Inage-ku, Chiba-shi, Chiba 263-8522, Japan. ${ }^{4}$ Department of Health Education and Health Sociology, School of Public Health, The University of Tokyo, 7-3-1 Hongo, Bunkyo-ku, Tokyo 113-0033, Japan. ${ }^{5}$ Department of Gerontological Evaluation, Center for Gerontology and Social Science, National Center for Geriatrics and Gerontology, 7-430 Morikoka-cho, Obu-shi, Aichi 474-8511, Japan. ${ }^{6}$ Department of Social Preventive Medical Sciences, Center for Preventive Medical Sciences, Chiba University, 1-8-1 Inohana, Chuo-ku, Chiba-shi, Chiba 260-8672, Japan. 
Received: 15 February 2018 Accepted: 9 October 2018

Published online: 19 October 2018

\section{References}

1. World Health Organization. World report on ageing and health. Geneva: World Health Organization; 2015.

2. National Institute of Population and Social Security Research. Household projections for Japan 2010-2035. National Institute of Population and Social Security Research. 2013. http://www.ipss.go.jp/pp-ajsetai/e/hhprj2013/tpage_e.asp. Accessed 1 Jun 2017.

3. Sharkey JR, Horel S, Dean WR. Neighborhood deprivation, vehicle ownership, and potential spatial access to a variety of fruits and vegetables in a large rural area in Texas. Int J Health Geogr. 2010;9:26.

4. Caspi CE, Sorensen G, Subramanian SV, Kawachi I. The local food environment and diet: a systematic review. Health Place. 2012;18(5):1172-87.

5. Satariano WA, Guralnik JM, Jackson RJ, Marottoli RA, Phelan EA, Prohaska TR. Mobility and aging: new directions for public health action. Am J Public Health. 2012;102(8):1508-15.

6. Banister D, Bowling A. Quality of life for the elderly: the transport dimension. Transp Policy. 2004;11(2):105-15.

7. Dean M, Raats MM, Grunert KG, Lumbers M. Factors influencing eating a varied diet in old age. Public Health Nutr. 2009;12(12):2421-7.

8. Glanz K, Johnson L, Yaroch AL, Phillips M, Ayala GX, Davis EL. Measures of retail food store environments and sales: review and implications for healthy eating initiatives. J Nutr Educ Behav 2016;48(4):280-8.e1.

9. Central Intelligence Agency. The world factbook. Central Intelligence Agency. 2017. https://www.cia.gov/library/publications/the-world-factbook/ index.html. Accessed 12 Jul 2017

10. Nakamura H, Nakamura M, Okada E, Ojima T, Kondo K. Association of food access and neighbor relationships with diet and underweight among community-dwelling older Japanese. J Epidemiol. 2017:27(11):546-51.

11. Sharkey JR, Johnson CM, Dean WR. Food access and perceptions of the community and household food environment as correlates of fruit and vegetable intake among rural seniors. BMC Geriatr. 2010;10:32.

12. Morland K, Filomena S. The utilization of local food environments by urban seniors. Prev Med. 2008:47(3):289-93.

13. Abusabha R, Namjoshi D, Klein A. Increasing access and affordability of produce improves perceived consumption of vegetables in low-income seniors. J Am Diet Assoc. 2011;111(10):1549-55.

14. Auchincloss AH, Diez Roux AV, Mujahid MS, Shen M, Bertoni AG, Carnethon MR. Neighborhood resources for physical activity and healthy foods and incidence of type 2 diabetes mellitus: the multi-ethnic study of atherosclerosis. Arch Intern Med. 2009;169(18):1698-704.

15. Cummins S, Macintyre S. Food environments and obesity — neighbourhood or nation? Int J Epidemiol. 2006;35(1):100-4.

16. Paquet C, Coffee NT, Haren MT, Howard NJ, Adams RJ, Taylor AW, et al. Food environment, walkability, and public open spaces are associated with incident development of cardio-metabolic risk factors in a biomedical cohort. Health Place. 2014;28:173-6.

17. McKinnon RA, Reedy J, Morrissette MA, Lytle LA, Yaroch AL. Measures of the food environment: a compilation of the literature, 1990-2007. Am J Prev Med. 2009:36(Suppl 4):S124-33.

18. Charreire H, Casey R, Salze P, Simon C, Chaix B, Banos A, et al. Measuring the food environment using geographical information systems: a methodological review. Public Health Nutr. 2010;13(11):1773-85.

19. Caspi CE, Kawachi I, Subramanian SV, Adamkiewicz G, Sorensen G. The relationship between diet and perceived and objective access to supermarkets among low-income housing residents. Soc Sci Med. 2012;75(7):1254-62.

20. Tani Y, Kondo N, Nagamine Y, Shinozaki T, Kondo K, Kawachi I, et al. Childhood socioeconomic disadvantage is associated with lower mortality in older Japanese men: the JAGES cohort study. Int J Epidemiol. 2016;45(4):1226-35.

21. Tani $Y$, Kondo N, Noma H, Miyaguni $Y$, Saito M, Kondo K. Eating alone yet living with others is associated with mortality in older men: the JAGES cohort survey. J Gerontol B Psychol Sci Soc Sci. 2017. https://doi.org/10. 1093/geronb/gbw211

22. Katz S, Downs TD, Cash HR, Grotz RC. Progress in development of the index of ADL. Gerontologist. 1970;10(1):20-30.

23. Industrial Statistics Office, Research and Statistics Department, Economic and Industrial Policy Bureau, Ministry of Economy, Trade and Industry. The Census of Commerce in 2007. http://www.meti.go.jp/english/statistics/. Accessed 20 Sep 2017.
24. Thornton LE, Pearce JR, Macdonald L, Lamb KE, Ellaway A. Does the choice of neighbourhood supermarket access measure influence associations with individual-level fruit and vegetable consumption? A case study from Glasgow. Int J Health Geogr. 2012;11:29.

25. Hanibuchi T, Nakaya T, Yonejima M, Honjo K. Perceived and objective measures of neighborhood walkability and physical activity among adults in Japan: a multilevel analysis of a nationally representative sample. Int J Environ Res Public Health. 2015;12(10):13350-64.

26. Tani Y, Sasaki Y, Haseda M, Kondo K, Kondo N. Eating alone and depression in older men and women by cohabitation status: the JAGES longitudinal survey. Age Ageing. 2015:44(6):1019-26.

27. Tani Y, Kondo N, Takagi D, Saito M, Hikichi H, Ojima T, et al. Combined effects of eating alone and living alone on unhealthy dietary behaviors, obesity and underweight in older Japanese adults: results of the JAGES. Appetite. 2015:95:1-8.

28. World Health Organization. Obesity: preventing and managing the global epidemic. Geneva: World Health Organization; 2000.

29. Matsuo T, Sairenchi T, Iso H, Irie F, Tanaka K, Fukasawa N, et al. Age- and gender-specific BMI in terms of the lowest mortality in Japanese general population. Obesity (Silver Spring). 2008;16(10):2348-55.

30. Wada T, Ishine M, Kita T, Fujisawa M, Matsubayashi K. Depression screening of elderly community-dwelling Japanese. J Am Geriatr Soc. 2003:51(9):1328-9.

31. Tani Y, Fujiwara T, Kondo N, Noma H, Sasaki Y, Kondo K. Childhood socioeconomic status and onset of depression among Japanese older adults: the JAGES prospective cohort study. Am J Geriatr Psychiatry. 2016; 24(9):717-26.

32. Fujiwara T, Takamoto I, Amemiya A, Hanazato M, Suzuki N, Nagamine Y, et al. Is a hilly neighborhood environment associated with diabetes mellitus among older people? Results from the JAGES 2010 study. Soc Sci Med. 2017;182:45-51.

33. Moore LV, Diez Roux AV, Nettleton JA, Jacobs DR Jr. Associations of the local food environment with diet quality - a comparison of assessments based on surveys and geographic information systems: the multi-ethnic study of atherosclerosis. Am J Epidemiol. 2008;167(8):917-24.

34. Matsuura T, Motohashi K. Market dynamics and productivity in Japanese retail industry in the late 1990s. Research Institute of Economy, Trade and Industry Discussion Paper Series 05-E-001. 2005. https://www.rieti.go.jp/jp/ publications/dp/05e001.pdf. Accessed 7 Jul 2018.

35. International Association of Traffic and Safety Sciences. White paper on traffic safety in Japan. 2011. http://www8.cao.go.jp/koutu/taisaku/h23kou_ haku/zenbun/genkyo/h1/h1b1s2_3.html. (in Japanese).

36. GF Ltd. Survey of dietary habits in older adults. 2011. http://www.seniorpromo.com/wordpress/wp-content/uploads/2011/02/senior_food.pdf. Accessed 15 Feb 2018. (in Japanese).

37. Fleischhacker SE, Evenson KR, Sharkey J, Pitts SB, Rodriguez DA. Validity of secondary retail food outlet data. A systematic review Am J Prev Med. 2013; 45(4):462-73.
Ready to submit your research? Choose BMC and benefit from:

- fast, convenient online submission

- thorough peer review by experienced researchers in your field

- rapid publication on acceptance

- support for research data, including large and complex data types

- gold Open Access which fosters wider collaboration and increased citations

- maximum visibility for your research: over $100 \mathrm{M}$ website views per year

At $\mathrm{BMC}$, research is always in progress.

Learn more biomedcentral.com/submission 